\title{
Static Magnetic Hyperfine Fields in Magnetically Polarized Pd
}

\author{
H. H. Bertschat, H.-H. Blaschek, H. Granzer, K. Potzger, S. Seeger, and W.-D. Zeitz \\ Bereich Festkörperphysik, Hahn-Meitner-Institut Berlin GmbH, D 14109 Berlin, Germany \\ H. Niehus \\ Humboldt-Universität zu Berlin, Institut für Physik, D 10115 Berlin, Germany
}

\author{
A. Burchard, D. Forkel-Wirth, and ISOLDE Collaboration \\ PPE Division, CERN, CH 1211, Genève 23, Switzerland
}

(Received 30 September 1997)

\begin{abstract}
In magnetically polarized ultrathin Pd films with (111) orientation, grown on a $\mathrm{Ni}(001)$ single crystal, static magnetic hyperfine fields were measured at the interface using perturbed angular correlation spectroscopy. The structural arrangement of the $\mathrm{Pd}$ atoms with respect to the Ni substrate differs from one $\mathrm{Pd}$ atom to the next, leading to varying $3 d-4 d$ electron hybridizations. This gives rise to a broad distribution of magnetic hyperfine fields at $\mathrm{Rh}$ nuclei, seen in experiments with the self-element probe ${ }^{100} \mathrm{Pd} /{ }^{100} \mathrm{Rh}$, while discrete field values were found for the voluminous impurity probe ${ }^{111} \mathrm{Cd}$. [S0031-9007(98)05565-3]
\end{abstract}

PACS numbers: 75.70.-i, 73.20.Hb, 76.80.+y

Magnetic interactions in $4 d$-electron elements (e.g., Ru, $\mathrm{Rh}, \mathrm{Pd})$ in contact with ferromagnetic $3 d$-electron elements $(\mathrm{Fe}, \mathrm{Co}, \mathrm{Ni})$ are the subject of numerous investigations [1-4]. Theoretical approaches for selected $3 d-4 d$ element systems are available (e.g., [5,6]). Because of its importance, the influence of structural properties on magnetic characteristics in ultrathin-layer systems is of high interest (e.g., [7-9]). Induced magnetic moments in $4 d$ element layers were found only in the immediate neighborhood of the interface, but recently it was shown that $\mathrm{Ni}$ induces fluctuating magnetic moments in bulk $\mathrm{Pd}$ up to at least 7 monolayers (ML) away from the interface [10].

The present experiment was undertaken to investigate the formation of static magnetic moments in thin layers of Pd (with deviating lattice parameters) evaporated on a ferromagnetic substrate. In particular, the measurementwith submonolayer resolution - of magnetic hyperfine fields $\left(B_{\mathrm{hf}}\right)$ in ultrathin Pd grown on a single crystal of $\mathrm{Ni}$ in correlation with the influence of local structural properties is the subject of this work. We used radioactive atoms as probes $\left({ }^{100} \mathrm{Pd},{ }^{111 m} \mathrm{Cd}\right.$ [11], experimental details in [10]) and applied the perturbed angular correlation spectroscopy (PAC) for the measurement of combined electric and magnetic hyperfine interactions. The PAC time spectra were measured in a 4-detector array and the interaction frequencies were deduced from the ratio function $R(t)$ of the count rates $C$ (angles between the detectors in degrees), given as

$$
R(t)=2\left[C\left(180^{\circ}\right)-C\left(90^{\circ}\right)\right] /\left[C\left(180^{\circ}\right)+C\left(90^{\circ}\right)\right] \text {. }
$$

All experiments were performed in the UHV chamber ASPIC (Apparatus for Surface Physics and Interfaces at CERN [10]) with a base pressure of $2 \times 10^{-9} \mathrm{~Pa}$. No contamination by the residual gas was detected by AES (Auger electron spectroscopy) during the measuring times

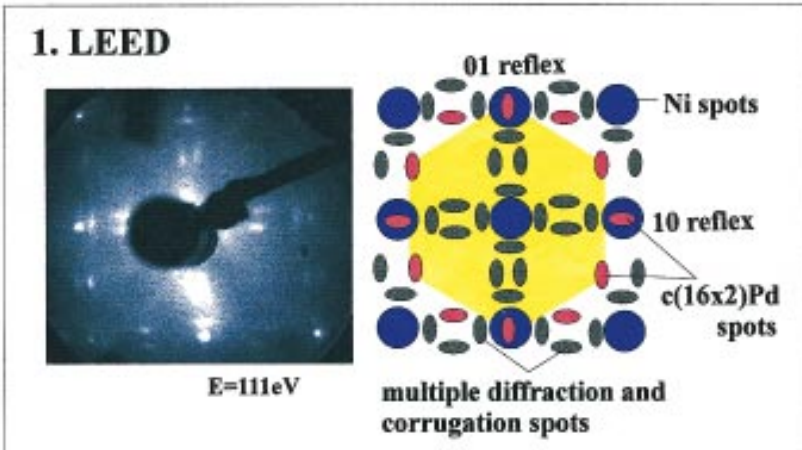

2. Model of $\mathrm{Ni}(001) \mathrm{c}(16 \mathrm{x} 2) \mathrm{Pd}, 1 / 2$ unit cell

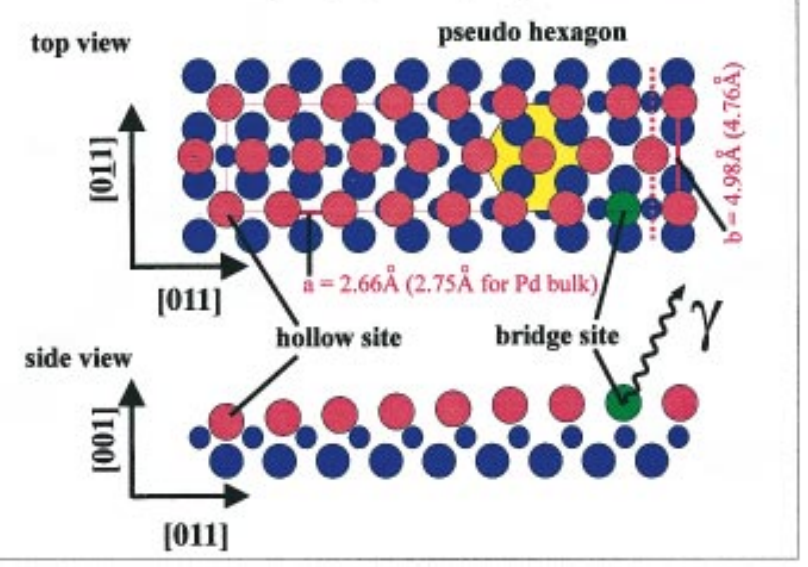

FIG. 1(color). LEED investigation of a single monolayer $\mathrm{Pd}$ on $\mathrm{Ni}(001)$; (top) LEED as observed and schematic representation. (center) First half of a $c(16 \times 2)$ unit cell $\mathrm{Pd}$ (red) on $\mathrm{Ni}(001)$ (blue) with a pseudohexagonal structure (yellow array). The product $a \times b=2.66 \times 4.98=13.2 \AA^{2}$ approaches the value for bulk $\operatorname{Pd}(111)$ : $13.1 \AA^{2} ; a(\mathrm{Ni})=$ $2.49 \AA$. Inserted is the radioactive impurity ${ }^{111 m} \mathrm{Cd}$ (green), its position is deduced from the PAC measurements. (bottom) A qualitative corrugation of the Pd coverage within the unit cell. 
for the PAC spectra, $12-24 \mathrm{~h}$ for ${ }^{100} \mathrm{Pd}\left(T_{1 / 2}=3.7 d\right)$ and $2-3 \mathrm{~h}$ for ${ }^{111 \mathrm{~m}} \mathrm{Cd}\left(T_{1 / 2}=49 \mathrm{~m}\right)$, where $2-3$ activation cycles were necessary for each of the spectra in Fig. 3.

In a first experimental step we determined the structure of Pd layers grown by molecular-beam epitaxy (MBE) on single crystals of Ni(001) [12]. The LEED investigation revealed that $\mathrm{Pd}$ grows with (111) orientation on $\mathrm{Ni}(001)$ (details in Fig. 1, lattice parameters in Table I).

In the next step we applied the self-element probe ${ }^{100} \mathrm{Pd}$ (decaying to ${ }^{100} \mathrm{Rh}$ ) in a PAC experiment for an investigation of local magnetic properties in one ML $\mathrm{Pd}$ on $\mathrm{Ni}$. In order to differentiate between magnetic and electric hyperfine interactions, we first performed a PAC experiment with ${ }^{100} \mathrm{Pd}$ in the topmost atomic layer of a (nonmagnetic) single crystal of Pd with (111) orientation. The electric hyperfine interaction frequency $\omega_{Q}=e Q V_{z z} /[4 I(2 I-1) \hbar]$ is essentially given as the product of the nuclear quadrupole moment $|Q|=0.151 \mathrm{~b}$ [10] and the electric field gradient (EFG) $V_{z z}$ arising from the nonsymmetric environment at the probe in the surface.

In Fig. 2(A), the ratio function of ${ }^{100} \mathrm{Pd} /{ }^{100} \mathrm{Rh}$ in the topmost layer of $\operatorname{Pd}(111)$ is shown. This is the first measurement of $\omega_{Q}\left({ }^{100} \mathrm{Rh}\right)$ in a surface layer. Fitting $\omega_{Q}$ to the data of Eq. (1), the quadrupole coupling constant was determined as $\nu_{Q}=e Q V_{z z} / h=22.3(2) \mathrm{MHz}$ [leading to $\left|V_{z z}\right|=6.1(2) \times 10^{17} \mathrm{~V} \mathrm{~cm}^{-2}$ at $\left.T=295 \mathrm{~K}\right]$ with an asymmetry parameter $\eta=0$ and the $z$ axis of the EFG pointing in the direction of the surface normal.

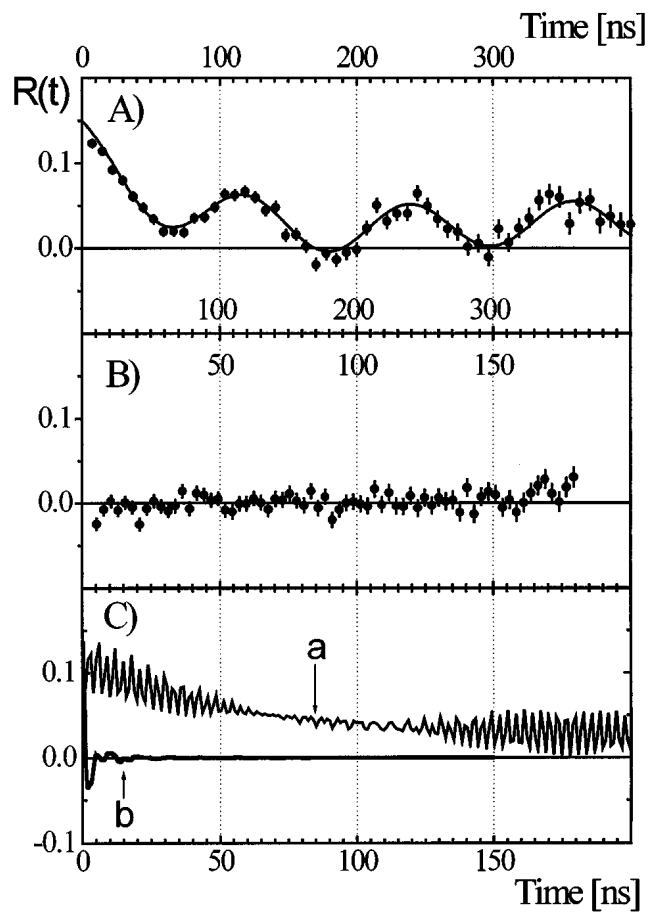

FIG. 2. PAC time spectra of ${ }^{100} \mathrm{Rh}$ (A) in the topmost layer of a single crystal of $\mathrm{Pd}(111)$; the data are fitted with $\omega_{Q}$ only, (B) in the adlayer of $\operatorname{Pd}(111)$ on $\mathrm{Ni}(001)$, different time scale. (C) Simulations, taking the EFG as measured in (A), combined (curve $a$ ) with $B_{\mathrm{hf}}=10 T$ ( $\frac{1}{2}$ of $B_{\mathrm{hf}}$ for ${ }^{100} \mathrm{Pd}$ in Ni [14]); (curve $b$ ) with a broad distribution of $B_{\mathrm{hf}} s$ around $5 \mathrm{~T}$.
Incorporating radioactive ${ }^{100} \mathrm{Pd}$ atoms into the single ML of Pd with (111) orientation on a single crystal of $\mathrm{Ni}(001)$, a similar - but not identical- $\omega_{Q}\left({ }^{100} \mathrm{Rh}\right)$ should contribute to the PAC time spectrum. Along with the additional magnetic hyperfine interaction with the Larmor frequency $\omega_{L}=g_{N} \mu_{N} B_{\mathrm{hf}} / \hbar$ ( $g_{N}$ nuclear $g$-factor, $\mu_{N}$ nuclear magneton; no external fields were applied) a combined interaction frequency $\omega_{c}=\omega_{c}\left(\omega_{Q}, \omega_{L}\right)$ [13] is expected. However, in the ${ }^{100} \mathrm{Rh}$ PAC time spectrum of our experiment [Fig. 2(B)] the amplitude of the frequencies disappeared within a few nanoseconds. We like to rule out that this is a result of a strong $B_{\mathrm{hf}}$ value in conflict with the time resolution, since the experiment was designed to measure values up to $30 \mathrm{~T}$, which is $150 \%$ of the value measured for Pd in Ni bulk [14]. The explanation of this effect can be deduced from a closer inspection of Fig. 1. Any $\mathrm{Pd}$ atom in comparison with any neighboring $\mathrm{Pd}$ atom has a different structural arrangement with respect to the $\mathrm{Ni}$ atoms. Consequently, the hybridization of the $3 d-4 d$ electrons, responsible for the magnetic polarization of $\mathrm{Pd}$, will be different from one Pd to the next. This results in a multitude of different $B_{\mathrm{hf}}$ values at the Pd sites within the unit cell. As a self-element atom, ${ }^{100} \mathrm{Pd}$ unselectively occupies any site of the unit cell; statistically, there is one probe atom per 30 unit cells. The spectrum in Fig. 2(B) hence reflects a broad distribution of (strong) static $B_{\mathrm{hf}} s$ in combination with (weak) electric interactions. In Fig. 2(C) some simulations of $\omega_{c}$ are shown. A comparison of the two experiments on $\mathrm{Pd}(111)$ and on $\mathrm{Ni}(001)-c(16 \times 2) \mathrm{Pd}$ leads to the conclusion that a single ML of Pd on Ni(001) is magnetically polarized with a local variation of induced static magnetic moments. The remote possibility that the result of the ${ }^{100} \mathrm{Pd}$ experiment, shown in Fig. 2(B), might be caused by fluctuating $B_{\mathrm{hf}} s$ as in bulk Pd [10], could definitely be excluded by the following experiment.

We chose the metallic impurity ${ }^{111 m} \mathrm{Cd} /{ }^{111} \mathrm{Cd}$ as a PAC probe [15] which was delivered by the mass separator ISOLDE/CERN [16]. The results of the ${ }^{111} \mathrm{Cd}$ PAC measurements are summarized in Table I and the corresponding time spectra are displayed in Fig. 3.

First, ${ }^{111 m} \mathrm{Cd}$ was incorporated into the topmost layer of $\mathrm{Ni}(001)$ by evaporating $\frac{1}{3} \mathrm{ML} \mathrm{Ni}$ on a single crystal by MBE techniques, consecutively adding $10^{-4} \mathrm{ML}$ of the radioactive probes, and then completing the ML Ni by MBE, all processed at $295 \mathrm{~K}$. The same procedure was repeated with MBE of $\mathrm{Pd}$ on the Ni substrate.

The data [of Eq. (1)] for Ni(001) were fitted [Fig. 3(A), solid line] including combined interaction frequencies $\omega_{c}$ and allowing for several fractions of probes at different positions [13]. The data could be fitted by only two fractions [separately shown in Figs. 3(A1) and 3(A2)]: Substitutional terrace-site and step-site occupations, respectively, which are identified by the EFG parameters (Table I). Our results confirm earlier measurements [17] and yield additional data.

For $\mathrm{Ni}(001)-c(16 \times 2) \mathrm{Pd}$ we found similar results. The $B_{\mathrm{hf}} s(\mathrm{Cd})$ are in plane as in $\mathrm{Ni}(001)$. In a remarkable 
TABLE I. Hyperfine-interaction parameters from ${ }^{111} \mathrm{Cd}$ PAC measurements.

\begin{tabular}{|c|c|c|c|c|}
\hline $\begin{array}{c}\text { System }^{\mathrm{a}} \\
\text { (Lattice parameter }[\AA])\end{array}$ & $\begin{array}{l}\text { Fraction }^{\mathrm{b}} \\
\qquad \%)\end{array}$ & $\begin{array}{c}\beta^{\mathrm{c}} \\
(\mathrm{deg})\end{array}$ & $\begin{array}{c}\left|V_{z z}\right|^{\mathrm{d}} \\
\left(10^{17} \mathrm{~V} \mathrm{~cm}^{-2}\right)\end{array}$ & $\begin{array}{c}\left|B_{\mathrm{hf}}\right| \\
(\mathrm{T})\end{array}$ \\
\hline $\mathrm{Ni}$ bulk ${ }^{\mathrm{e}}$ (2.49) & $>50$ & $\ldots$ & $\ldots$ & $7.2(1)$ \\
\hline \multirow[t]{2}{*}{ Topmost layer Ni } & terrace 15 & 0 & $8.2(2)^{f}$ & $3.5(4)^{f}$ \\
\hline & step 25 & $35(8)$ & $7.3(3)$ & $3.9(4)$ \\
\hline $0.5 \mathrm{ML} P d(2.66)^{\mathrm{g}}$ & step 70 & $54(4)$ & $7.3(3)$ & $3.3(3)$ \\
\hline \multirow[t]{2}{*}{$1 \mathrm{ML} \operatorname{Pd}(2.66)^{g}$} & terrace 15 & 0 & $10.9(2)^{h}$ & $3.9(3)$ \\
\hline & step 20 & $58(8)$ & $7.4(2)^{\mathrm{i}}$ & $3.3(3)$ \\
\hline Topmost layer of & terrace 50 & 0 & $10.6(3)^{h}$ & $1.2(4)$ \\
\hline $2 \mathrm{ML} \operatorname{Pd}(2.70)^{\mathrm{h}}$ & step 10 & $58(8)$ & $7.5(3)^{\mathrm{i}}$ & $3(1)$ \\
\hline
\end{tabular}

${ }^{\mathrm{a}}$ All measurements are performed on $\mathrm{Ni}(001)$ between 270 and $300 \mathrm{~K}$.

${ }^{b}$ Listed are the fractions of probes with well-defined hyperfine-interaction frequencies; the rest $(\approx 50 \%)$ is positioned at imperfections with a broad distribution of $V_{z z}$ and $B_{\mathrm{hf}}$ values. Best layer growth of Pd (quality of LEED spectra) was obtained at $270 \mathrm{~K}$ (explored range: $100-400 \mathrm{~K}$ ).

${ }^{\mathrm{c}}$ Angle of the EFG deviating from the surface normal.

${ }^{\mathrm{d}}$ With $Q\left({ }^{111} \mathrm{Cd}\right)=0.83 \mathrm{~b}[11]$.

'Taken from [20], $T \rightarrow 0 \mathrm{~K}$.

fIn agreement with [17].

gIn [0-11] direction the Pd coverage adopts the lattice parameter of $\mathrm{Ni}$.

${ }^{\mathrm{h}}$ Approaching the Pd bulk lattice parameter $(2.75 \AA)$, the $V_{z z}$ values for the terrace positions approach the value $\left|V_{z z}\right|=10.2(2) \times$ $10^{17} \mathrm{~V} \mathrm{~cm}^{-2}$ measured for a single crystal $\mathrm{Pd}(111)$ [15].

in agreement with nonsubstitutional step sites [15]; values are less dependent on the lattice parameters.

contrast to our experiment with ${ }^{100} \mathrm{Pd}$, here we obtained discrete values: $\left|B_{\mathrm{hf}}(\mathrm{Cd})\right|=3.9(3) \mathrm{T}$ for the occupation within the terrace and $\left|B_{\mathrm{hf}}(\mathrm{Cd})\right|=3.3(3) \mathrm{T}$ for the (nonsubstitutional) step-site position [18]. Within the time window, given by the half-life of the nuclear state, almost no damping of the amplitude is observed, indicating that the probes are located at sites with almost no lattice disturbances. Either the $s p$-element $\mathrm{Cd}$ senses the same $B_{\mathrm{hf}}$ at any position in the Pd layer or the incorporation of an impurity in $\mathrm{Ni}(001)-c(16 \times 2) \mathrm{Pd}$ results in a selected occupation of preferential or even uniform sites within the unit cell. In Fig. 1, bottom, a possible corrugation of the Pd atoms is modeled qualitatively. Presumably, Cd prefers bridgelike sites rather than hollowlike sites because of its larger size. Its high mobility at surfaces (at $295 \mathrm{~K}$ ) [15] enables $\mathrm{Cd}$ to occupy such sites during the process of the sample preparation.

The separation of the two fractions [substitutional terrace-site occupation, Fig. 3(B1) and step-site occupation, 3(B2)] could easily be checked by growing $0.5 \mathrm{ML}$ of $\mathrm{Pd}$ on $\mathrm{Ni}(001)$. The LEED investigation showed the formation of unit cells with the same parameters and the same (111) orientation as was found for the complete ML Pd. However, at this fractionally covered surface, the number of step positions available for the probe atoms had increased tremendously, and the majority of ${ }^{111} \mathrm{Cd}$ probes occupied step sites as reflected in the combined interaction frequency [Fig. 3(C)], already found in Fig. 3(B2). The spectrum shows a stronger damping, which is expected considering the roughness: Slight differences in the island growth cause a slight distribution of $B_{\mathrm{hf}} \mathrm{S}$ and EFGs. It might be interesting to note that the $B_{\mathrm{hf}}$ values for both step positions [Figs. 3(B2) and 3(C)] are indistinguishable, although the immediate local environments are different. Incorporating the probe atoms into the topmost of two ML Pd on Ni(001) [Fig. 3(D), [18] ], a considerably reduced field, $\left|B_{\mathrm{hf}}\right|=1.2(4) \mathrm{T}$, was measured for the substitutional terrace position.

Within this study we present the first measurements of static magnetic hyperfine fields in ultrathin films of the strongly exchange-enhanced $4 d$-element Pd. The existence of these fields can be understood as a consequence of the induced magnetic order in Pd. In the first experiments on the Ni/Pd layer system [19], the formation of static moments was anticipated, meanwhile, however, it was shown for thicker Pd systems that the induced magnetic moments become unstable [10]. The absolute value of $B_{\mathrm{hf}}$ at ${ }^{111} \mathrm{Cd}$ in the $\mathrm{Pd}$ adlayer is tentatively even stronger as compared with the value in the topmost layer of $\mathrm{Ni}$, indicating that the magnetic polarization is rather strong in the isoelectric Pd layer with direct contact to Ni. At a comparable position in the $\mathrm{Ni} / \mathrm{Cu}$ system $B_{\mathrm{hf}}$ is zero [20]. The strong polarization in the adlayer propagates the polarization into the second Pd layer with a smaller, although still measurable value. Although at present no calculations are available for magnetic hyperfine fields in spin polarized Pd in contact with $\mathrm{Ni}$, we can compare our results with theoretical estimates for the gradient from atomic layer to atomic layer of induced magnetic moments in Pd calculated for a Fe/Pd system. For the second atomic layer the moments decrease to $\approx 30 \%$ [5]; our study quantitatively substantiates these predictions for terrace positions.

Using the different characteristics of the self-element ${ }^{100} \mathrm{Pd}$ and the impurity ${ }^{111 m} \mathrm{Cd}$ as probes, we were able to 


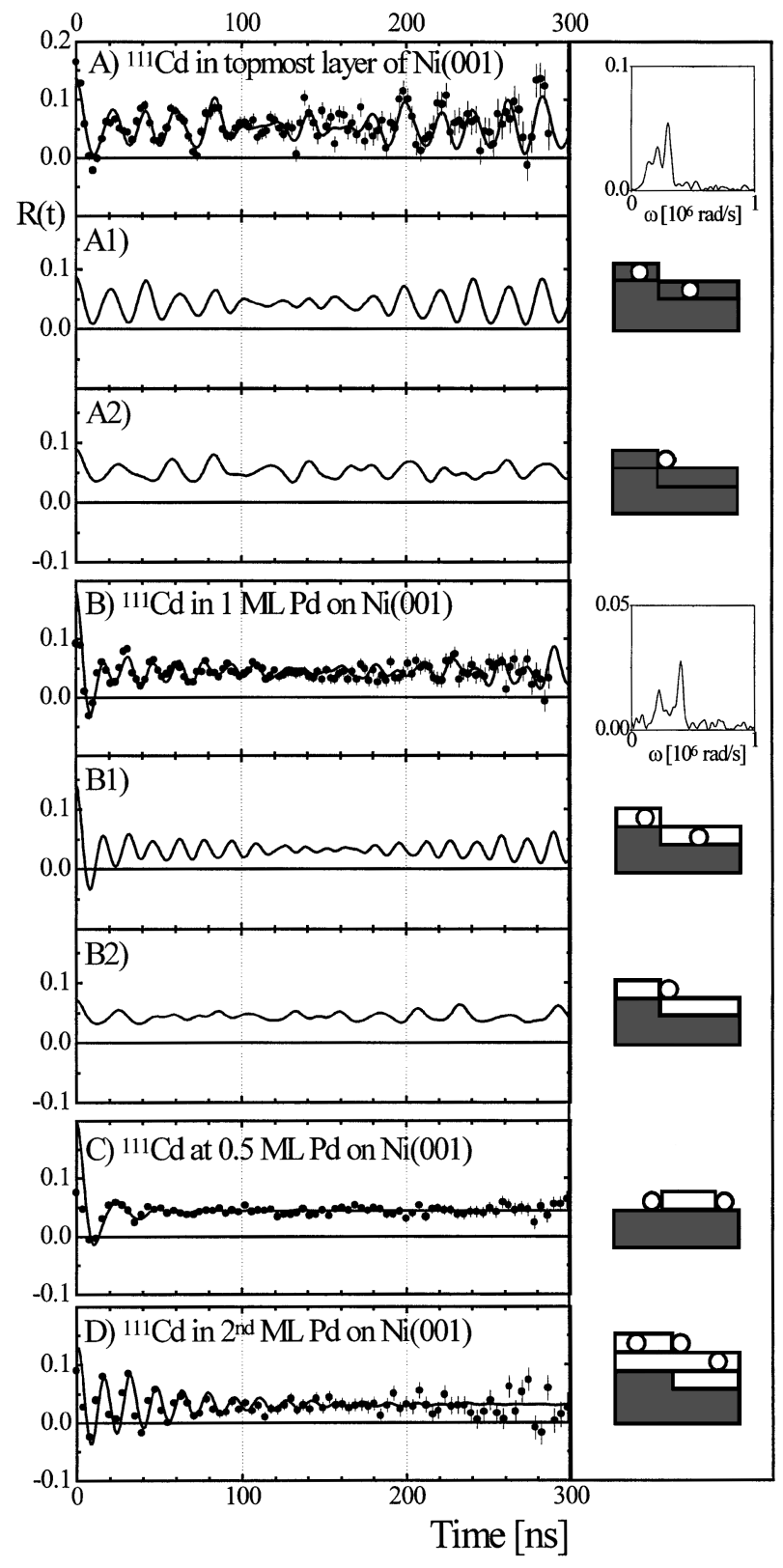

FIG. 3. PAC spectra of ${ }^{111} \mathrm{Cd}$ (positions of the probes are shown in the sketches): (A) in the topmost layer of $\mathrm{Ni}(001)$, Fourier transformation on the right; (B) in one ML of $\mathrm{Pd}(111)$ on $\mathrm{Ni}(001)$; (C) at steps for 0.5 ML Pd on Ni(001); (D) in the topmost layer of two ML Pd on Ni(001). The fits to the data in (A) and (B) are composed of the fractions for terrace atoms (A1) and (B1), respectively; and for step atoms (A2) and (B2), respectively.

look - on an atomic scale - into the intimate correlation between local magnetic and structural properties at the $\mathrm{Ni} /$ Pd interface.

We thank J. Lohmüller and G. Schatz for support in the early stages of the experiments, H. Haas and R. Kowallik for technical assistance, and D. Riegel for discussion. This work was supported by resources of the Kernforschungszentrum Karlsruhe $\mathrm{GmbH}$. The ${ }^{100} \mathrm{Pd}$ nuclide production was conducted by K.-H. Assmus/ Karlsruhe.
[1] B. Heinrich and J.F. Cochran, Adv. Phys. 42, 523 (1993).

[2] U. Gradmann in Handbook of Magnetic Materials, edited by K. H. J. Buschow (Elsevier, Amsterdam, 1993), Vol. 7, p. 1.

[3] A. Rampe, D. Hartmann, W. Weber, S. Popovic, M. Reese, and G. Güntherodt, Phys. Rev. B 51, 3230 (1995).

[4] K. Totland, P. Fuchs, J. C. Gröbli, and M. Landolt, Phys. Rev. Lett. 70, 2487 (1993).

[5] P. H. Dederichs, P. Lang, K. Willenborg, R. Zeller, N. Papanikolaou, and N. Stefanou, Hyperfine Interact. 78, 341 (1993).

[6] C. L. Fu, A. J. Freeman, and T. Oguchi, Phys. Rev. Lett. 54, 2700 (1985); J. Mathon, J. Phys. F 16, 669 (1986); R. E. Camley and R. L. Stamps, J. Phys. Condens. Matter 5, 3727 (1993).

[7] U. Gradmann, T. Dürkop, and H.J. Elmers, J. Magn. Magn. Mater. 165, 56 (1997).

[8] E.E. Fullerton, D. Stoeffler, K. Ounadjela, B. Heinrich, Z. Celinsky, and J.A.C. Bland, Phys. Rev. B 51, 6364 (1995).

[9] M. Wuttig, B. Feldmann, and T. Flores, Surf. Sci. 331333, 659 (1995).

[10] H.H. Bertschat, H. Granzer, H. Haas, R. Kowallik, S. Seeger, and W.-D. Zeitz, Phys. Rev. Lett. 78, 342 (1997). Ref. [17] refers to the present experiment with a preliminary PAC spectrum in Fig. 2(c).

[11] Table of Isotopes, edited by R. B. Firestone and V.S. Shirley (Wiley \& Sons, New York, 1996), 8th ed.; R. Vianden, Hyperfine Interact. 15/16, 1081 (1983).

[12] The crystals were cut and polished by U. Linke, IGV at KfK Jülich, Germany.

[13] All PAC spectra with combined interactions were evaluated using the code developed by B. Lindgren, Hyperfine Interact. (C) 1, 613 (1996).

[14] S. Koički, T. A. Koster, R. Pollak, D. Quitmann, and D. A. Shirley, Phys. Lett. 32B, 351 (1970).

[15] E. Hunger and H. Haas, Surf. Sci. 234, 273 (1990).

[16] E. Kugler et al., Nucl. Instrum. Methods Phys. Res., Sect. B 70, 41 (1992).

[17] J. Voigt, Ph.D. thesis, Universität Konstanz, 1990 (unpublished).

[18] In favorable cases-depending on $Q, V_{z z}, \mu, B_{\mathrm{hf}}$, etc. - the PAC spectra of combined interactions are dominated by two frequencies resulting in beat patterns falling into the time window given by the half-life of the nuclear state. By chance, this is the case for ${ }^{111} \mathrm{Cd}$ in the topmost layer of $\mathrm{Ni}$ [Fig. 3(A1)] and in one ML Pd on Ni [Fig. 3(B1)] where the frequency nodes are to be seen around $150 \mathrm{~ns}$. In Fig. 3(D) the node would occur at about $3 \mu \mathrm{s}$, far outside the time window because of the small $B_{\mathrm{hf}}$ (Table I). The damping of the amplitude is caused by a spread of the frequencies due to the less perfect structure of the 2nd ML of Pd.

[19] U. Gradmann and R. Bergholz, Phys. Rev. Lett. 52, 771 (1984).

[20] J. Voigt, X. L. Ding, R. Fink, G. Krausch, B. Luckscheiter, R. Platzer, U. Wöhrmann, and G. Schatz, Phys. Rev. Lett. 66, 3199 (1991). 